\title{
Flots topologiquement transitifs sur les surfaces compactes sans bord: contrexemples à une conjecture de Katok
}

\author{
GILBERT LEVITT \\ Département de Mathématiques, Université Paris VII, 2 Place Jussieu, \\ 75251 Paris, Cedex 05, France
}

(Received 2 June 1982 and revised 15 September 1982)

Abstract. We prove that on closed surfaces of higher genus cohomological invariants are not sufficient to distinguish topologically transitive flows which are not topologically conjugate; this contradicts a conjecture of Katok.

\section{Introduction}

Soit sur une surface fermée orientée $M$ de genre $g \geq 1$ un champ de vecteurs $X$ possédant une orbite récurrente. L'exemple le plus simple est un 'flot irrationnel' sur $T^{2}$, et plus généralement un champ sur $M$ possédant une orbite dense (un tel champ sera dit transitif).

En fait l'étude dynamique et ergodique des orbites d'un champ quelconque se ramène à celle des champs transitifs $[13],[6]$. Il est donc particulièrement important d'essayer de classifier les champs transitifs sur une surface donnée $\boldsymbol{M}$. Pour cela on peut associer à un champ transitif $X$ un invariant de nature cohomologique, un cône convexe non vide $\grave{\mathscr{C}}(X)$ ne contenant pas 0 dans l'espace vectoriel $H^{1}(M, F ; \mathbb{R})$ $(F=F(X)$ désigne l'ensemble des singularités de $X$, supposé fini); nous définirons $\mathscr{C}(\boldsymbol{X})$ dans la section 1.

Katok a défini ce cône dans [3], et il a énoncé le théorème suivant, qui exprime que $\mathscr{C}(X)$ permet de classifier les champs $\boldsymbol{X}$ proches d'un champ fixe $\boldsymbol{X}_{0}$ :

THÉORÈME. Soient $X_{0}$ et $X$ deux champs transitifs à singularités non dégénérées (qui sont donc $2 g-2$ selles), tels que $F(X)=F\left(X_{0}\right)$ et que $\mathscr{C}(X) \cap \mathscr{C}\left(X_{0}\right)$ ne soit pas vide (donc contienne au moins une demi-droite); si $X$ est suffisamment proche de $X_{0}$, alors $X$ et $X_{0}$ sont topologiquement conjugués.

(Deux champs $X$ et $X_{0}$ sont topologiquement conjugués s'il existe un homéomorphisme envoyant chaque orbite de $X_{0}$ sur une orbite de $X$, sans nécessairement respecter les paramétrages; en d'autres termes, les feuilletages définis par $X$ et $X_{0}$ sont topologiquement conjugués.)

Katok a également conjecturé dans [3] que ce théorème reste vrai même si $X$ et $X_{0}$ ne sont pas proches. Le but du présent article est de montrer que cette conjecture est fausse. Plus précisément: 
THÉORÈME 1. Soit $M$ une surface fermée orientée de genre $g \geq 2$, et $F \subset M$ un ensemble formé de $2 g-2$ points. Pour presque toute classe de cohomologie $\Omega \in H^{1}(M, F ; \mathbb{R})$, il existe une infinité de champs de vecteurs $\left(X_{i}\right)_{i \geq 1}$ à singularités non dégénérées, tels que:

(a) pour tout $i$, les singularités de $X_{i}$ sont les points de $F$.

(b) pour tout $i$, toute orbite de $X_{i}$ qui n'est pas réduite à un point est dense.

(c) pour tout $i, \mathscr{C}(X)$ est égal à la demi-droite $\{t \Omega: t>0\}$.

(d) pour $i \neq j$, les champs $X_{i}$ et $X_{j}$ ne sont pas topologiquement conjugués.

Remarques. (1) La locution 'presque toute' se rapporte à une mesure de Lebesgue sur $H^{1}(M, F ; \mathbb{R})$; nous montrerons également que l'ensemble des classes $\Omega \in$ $H^{1}(M, F ; \mathbb{R})$ auxquelles s'applique le résultat du théorème 1 contient un $G_{\delta}$ dense (voir remarque après la démonstration du lemme 1 ).

(2) D'après le théorème de Katok énoncé plus haut, l'infinité de champs $X_{i}$ construite dans le théorème 1 est nécessairement dénombrable.

(3) Soit $\Omega \in H^{1}(M, F ; \mathbb{R})$ telle que la classe $\bar{\Omega} \in H^{1}(M ; \mathbb{R})$ associée à $\Omega$ soit multiple d'une classe entière. Le résultat du théorème 1 , même considérablement affaibli, ne s'applique pas à une telle classe (voir remarquè après l'énoncé du théorème 2).

Dans la situation du théorème de Katok, les champs $X$ et $X_{0}$ sont conjugués par un homéomorphisme proche de l'identité, et donc isotopes (conjugués par un homéomorphisme isotope à l'identité). On remarquera qu'il est facile de construire une infinité de champs transitifs possédant les mêmes singularités et le même $\mathscr{C}(X)$, mais non isotopes: par exemple deux champs à singularités non dégénérées, qui satisfont à (b) et différent par un (ou plusieurs) twists de Dehn le long d'une courbe fermée simple homologue à 0 , ne sont pas isotopes (utiliser [1, exposé 6 , $\S$ VII, corollaire p. 118 , et exposé 5, proposition II.6 p. 81]). Par contre prouver que deux champs ne sont pas conjugués est beaucoup plus délicat, et constitue toute la difficulté du théorème 1.

Décider si deux champs sont ou non conjugués fait intervenir la structure transverse des feuilletages déterminés par ces champs, beaucoup plus que le paramétrage des feuilles donné par les champs. C'est pourquoi pour prouver le théorème 1 nous raisonnerons avec des 1 -formes différentielles plutôt qu'avec des champs de vecteurs.

Notre première tâche sera donc, après avoir défini le cône $\mathscr{C}(X)$, de reformuler le théorème 1 en termes de formes différentielles. Nous consacrerons ensuite la section 2 à la démonstration, puis nous concluerons dans la section 3 par quelques exemples, remarques, et questions.

\section{Le cône $\mathscr{C}(X)$}

Nous allons définir ici le cône $\mathscr{C}(X) \subset H^{1}(M, F ; \mathbb{R})$. L'espace vectoriel $H^{1}(M, F ; \mathbb{R})$ est de dimension $4 g-3$. Il s'identifie canoniquement à $\operatorname{Hom}\left(H_{1}(M, F ; \mathbb{R}), \mathbb{R}\right)$. Nous considérerons souvent un élément $\Omega$ de $H^{i}(M, F ; \mathbb{R})$ comme une collection de 1 -formes différentielles fermées cohomologues relativement à $F$ (i.e. différant par la différentielle d'une fonction qui s'annule sur $F$ ); l'homomorphisme de 
$H_{1}(M, F ; \mathbb{R})$ dans $\mathbb{R}$ associé à $\Omega$ et alors déterminé par l'intégration de ces formes.

Nous supposons pour simplifier que les singularités de $X$ ne sont pas dégénérées et que toute orbite non réduite à un point est dense. Nous orientons transversalement le feuilletage $\mathscr{F}$ déterminé par $X$, de façon qu'un repère formé de $X$ et d'un vecteur positivement transverse soit direct.

Soit $\mu$ une mesure transverse non nulle de $\mathscr{F}$ (il en existe toujours). Etant donné un chemin orienté $\gamma$ formé d'un nombre fini de segments de feuilles de $\mathscr{F}$ et de segments $\gamma_{i}$ transverses à $\mathscr{F}$, on définit un nombre $(\mu)(\gamma)$ comme la somme des $\varepsilon_{i} \mu\left(\gamma_{i}\right)$, où $\varepsilon_{i}$ vaut +1 ou -1 selon que l'orientation de $\gamma_{i}$ coïncide ou non avec l'orientation transverse de $\mathscr{F}$.

On vérifie facilement que $(\mu)(\gamma)$ ne dépend que de la classe d'homotopie à extrémités fixes de $\gamma$; on peut donc déduire de $(\mu)$ un homomorphisme de $H_{1}(M, F ; \mathbb{R})$ dans $\mathbb{R}$, c'est à dire un élément $[\mu]$ de $H^{1}(M, F ; \mathbb{R})$. Le cône $\mathscr{C}(X)$ est défini comme l'ensemble des $[\mu] \in H^{1}(M, F ; \mathbb{R})$ correspondant aux diverses mesures transverses de $\mathscr{F}$. Il n'est pas vide et ne contient pas 0 .

Remarque. On peut définir $\mathscr{C}(\boldsymbol{X})$ de plusieurs autres façons équivalentes (comparer [2], [3], [10], [11]). En particulier la dualité de Lefschetz permet de considérer les éléments de $\mathscr{C}(X)$ comme des cycles asymptotiques dans $H_{1}(M-F ; \mathbb{R})$.

Supposons en particulier que $\mathscr{F}$ soit défini par une 1-forme différentielle fermée $\mathscr{W}$ (i.e. l'évaluation $\mathscr{W}(X)$ est identiquement nulle), et que $\mathscr{W}$ soit compatible avec l'orientation transverse de $\mathscr{F}$ (en ce sens que $\mathscr{W}(c)>0$ pour un vecteur $c$ positivement transverse à $\mathscr{F})$. La formule

$$
\mu_{W}(J)=\left|\int_{J} \mathscr{W}\right|
$$

où $J$ est un intervalle transverse à $\mathscr{F}$, définit une mesure transverse $\mu_{\mathscr{W}}$ de $\mathscr{F}$, qui est invariante parce que $\mathscr{W}$ est fermée. La classe $[\mu \mathscr{W}] \in H^{1}(M, F ; \mathbb{R})$ n'est autre que la classe de cohomologie relative de la forme fermée $\mathscr{W}$.

Avant de reformuler le théorème 1 , nous devons donner une dernière définition. Un feuilletage dont les singularités sont les points de $F$ est uniquement ergodique si sa restriction à $M-F$ possède, à proportionnalité près, une seule mesure transverse invariante; si un champ $X$ comme plus haut définit un feuilletage uniquement ergodique, alors $\mathscr{C}(\boldsymbol{X})$ se compose d'une demi-droite. Il est maintenant clair que le théorème 1 est une conséquence du théorème suivant:

THÉORÈME 2. Soient $M$ et $F$ comme dans l'énoncé du théorème 1. Presque toute classe de cohomologie $\Omega \in H^{1}(M, F ; \mathbb{R})$ contient une infinité de 1-formes différentielles fermées $\left(\mathscr{W}_{i}\right)_{i \geq 1}$ à singularités non dégénérées définissant des feuilletages $\mathscr{F}\left(\mathscr{W}_{i}\right)$ tels que:

(a) pour tout $i$, les singularités de $\mathscr{F}\left(\mathscr{W}_{i}\right)$ sont les points de $F$.

(b) pour tout $i$, toute feuille de $\mathscr{F}\left(\mathscr{W}_{i}\right)$ est dense.

(c) pour tout $i$, le feuilletage $\mathscr{F}\left(\mathscr{W}_{i}\right)$ est uniquement ergodique.

(d) pour $i \neq j$, les feuilletages $\mathscr{F}\left(\mathscr{W}_{i}\right)$ et $\mathscr{F}\left(\mathscr{W}_{j}\right)$ ne sont pas topologiquement conjugués. 
Remarque. Considérons une classe $\Omega \in H^{1}(M, F ; \mathbb{R})$ telle que la classe $\bar{\Omega} \in H^{1}(M ; \mathbb{R})$ associée à $\Omega$ soit un multiple d'une classe entière. Soit $\mathscr{W} \in \Omega$ une forme fermée à singularités non dégénérées satisfaisant à (a). Nous affirmons que toute feuille $f$ de $\mathscr{F}(\mathscr{W})$ qui n'est pas une liaison entre selles est compacte: sinon, $f$ possèderait un point d'accumulation et il existerait des intervalles transverses à extrémités sur $f$ sur lesquels l'intégrale de $\mathscr{W}$ est arbitrairement proche de 0 , ce qui contredit l'hypothèse faite sur $\bar{\Omega}$. De cette remarque on déduit facilement que les formes fermées à singularités non dégénérées contenues dans $\Omega$ ne définissent, à conjugaison topologique près, qu'un nombre fini de feuilletages.

\section{Démonstration du théorème 2}

Soient $M$ et $F$ comme dans l'énoncé du théorème 1. Pour simplifier, nous convenons que dorénavant le seul mot forme désignera une 1-forme différentielle fermée dont les singularités sont non dégénérées et coïncident avec $F$. Deux formes sont transverses si elles définissent des feuilletages transverses en dehors de $F$.

Soit $G$ le groupe des homéomorphismes $\psi$ de $M$ tels que $\psi(F)=F$. Pour $\psi \in G$, nous notons $\psi^{*}$ l'automorphisme induit sur $H^{1}(M, F ; \mathbb{R})$, et nous désignons par $G_{1}$ le groupe formé des $\psi \in G$ tels que $\psi^{*}=\varepsilon$ id, avec $\varepsilon= \pm 1$ (si $g \geq 3$, nécessairement $\varepsilon=+1$ ). Deux feuilletages sont $G_{1}$-conjugués s'ils sont conjugués par un homéomorphisme $\psi \in G_{1}$.

Notre but va être par des réductions successives de ramener le théorème 2 à un problème de nature algébrique dans $H_{1}(M ; \mathbb{Z})$. La première étape consiste à définir un ensemble $A \subset H^{1}(M, F ; \mathbb{R})$ de complémentaire négligeable, dans lequel la conclusion du théorème 2 sera valable.

Soit $A$ le sous-ensemble de $H^{1}(M, F ; \mathbb{R})$ formé des classes $\Omega$ telles que:

(i) l'homomorphisme de $H_{1}(M, F ; \mathbb{Z})$ dans $\mathbb{R}$ induit par $\Omega$ est injectif.

(ii) si $\psi^{*} \Omega=t \Omega$, avec $\psi \in G$ et $t \in \mathbb{R}$, alors $\psi \in G_{1}$.

(iii) si le feuilletage défini par une forme $\mathscr{W} \in \Omega$ ne possède pas de liaison séparante, alors il satisfait aux conditions (b) et (c) du théorème 2 (une liaison séparante est une feuille de $\mathscr{F}(\mathscr{W})$ qui joint une selle $s$ à elle-même et disconnecte $M-\{s\})$.

LeMME 1 Le complémentaire de $A$ dans $H^{1}(M, F ; \mathbb{R})$ est négligeable (pour une mesure de Lebesgue).

Démonstration. Nous laissons au lecteur le soin de vérifier que presque toute classe $\Omega \in H^{1}(M, F ; \mathbb{R})$ satisfait aux conditions (i) et (ii). Remarquons ensuite que, si une forme $\mathscr{W}$ appartient à une classe $\Omega$ qui vérifie (i), alors toute liaison de $\mathscr{F}(\mathscr{W})$ représente 0 dans $H_{1}(M, F ; \mathbb{Z})$, et donc est une liaison séparante. Il en résulte que, si $\mathscr{F}(\mathscr{W})$ ne possède pas de liaison séparante, toute feuille de $\mathscr{F}(\mathscr{W})$ est dense (voir [1, exposé $9, \S$ III, lemme 6 p. 165]). Il nous reste donc à exclure l'existence dans $H^{1}(M, F ; \mathbb{R})$ d'un ensemble $B$ de mesure strictement positive tel que toute classe $\Omega \in B$ contienne une forme $\mathscr{W}_{\Omega}$ avec $\mathscr{F}\left(\mathscr{W}_{\Omega}\right)$ non uniquement ergodique mais à feuilles denses. 
Etant donné $\Omega \in B$, choisissons une courbe fermée simple $C$ transverse à $\mathscr{F}\left(\mathscr{W}_{\Omega}\right)$, et considérons le 1-complexe fini $K_{\Omega}$ formé par $C$ et les séparatrices de $\mathscr{F}\left(\mathscr{W}_{\Omega}\right)$, arrêtées à leur premier point d'intersection avec $C$. Modulo une isotopie relative à $F$, l'ensemble des $K_{\Omega}$ possibles est dénombrable, et on peut donc supposer sans perte de généralité que $K_{\Omega}$ ne dépend pas de $\Omega$.

Le feuilletage $\mathscr{F}\left(\mathscr{W}_{\Omega}\right)$ s'identifie à la suspension d'un échange d'intervalles sur $C$, voir $[5, \S 6]$. Cet échange porte sur $4 \mathrm{~g}-4$ intervalles et dér $-\underset{d e}{-} 4 \mathrm{~g}-3$ paramétres réels (longueurs des intervalles et image d'un point); l'application qui à $\Omega$ associe l'ensemble de ces paramètres est (localement) linéaire et injective, et la contradiction cherchée résulte du fait que presque tout échange d'intervalles induisant une permutation irréductible donnée est uniquement ergodique, [7], [14]; voir aussi [9].

Remarque. Etre uniquement ergodique est une propriété générique des échanges d'intervalles ([4]; [3, théorème 4]). On en déduit que $A$ contient un $G_{\delta}$ dense.

Soit $\Omega \in A$. Pour pouvoir construire dans $\Omega$ des formes définissant des feuilletages sans liaison séparante mais non conjugués, nous aurons besoin du critère suivant:

Proposition. Soient $\mathscr{W}^{\text {et }} \mathscr{W}^{\prime}$ deux formes appartenant à une classe $\Omega \in A$. Soient

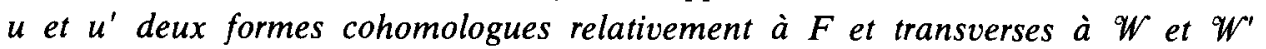
respectivement. Si $\mathscr{F}(u)$ et $\mathscr{F}\left(u^{\prime}\right)$ ne sont pas $G_{1}$-conjugués, alors $\mathscr{F}(\mathscr{W})$ et $\mathscr{F}\left(\mathscr{W}^{\prime}\right)$ ne sont pas conjugués.

Avant de prouver cette proposition, remarquons que l'existence de $u$ (resp. $u^{\prime}$ ) entraîne que $\mathscr{F}(\mathscr{W})$ (resp. $\mathscr{F}\left(\mathscr{W}^{\prime}\right)$ ) ne possède pas de liaison séparante; les feuilletages $\mathscr{F}(\mathscr{W})$ et $\mathscr{F}\left(\mathscr{W}^{\prime}\right)$ sont donc uniquement ergodiques et à feuilles denses.

Nous appliquerons ce critère avec des formes $u$ et $u^{\prime}$ appartenant à une classe de cohomologie entière $U \in H^{1}(M, F ; \mathbb{Z})$. De telles formes définissent des feuilletages à feuilles compactes (voir fin de la section 1), donc faciles à comprendre. En particulier il est très simple de décider si $\mathscr{F}(u)$ et $\mathscr{F}\left(u^{\prime}\right)$ sont ou non $G_{1}$-conjugués, et de prouver que les formes de $U$ définissent une infinité de feuilletages à $G_{1}$-conjugaison près (bien qu'elles définissent un nombre fini de feuilletages à conjugaison près).

Démonstration de la proposition. Supposons qu'un homéomorphisme $\psi$ conjugue $\mathscr{F}(\mathscr{W})$ et $\mathscr{F}\left(\mathscr{W}^{\prime}\right)$. Il envoie la mesure transverse $\mu \mathscr{W}$ de $\mathscr{F}(\mathscr{W})$ sur une mesure transverse de $\mathscr{F}\left(\mathscr{W}^{\prime}\right)$, qui s'écrit nécessairement $r . \mu_{W^{\prime}}$ (car $\mathscr{F}\left(\mathscr{W}^{\prime}\right)$ est uniquement ergodique). Il existe donc une relation de la forme

$$
\psi^{*} \Omega=\varepsilon r \Omega
$$

(le signe de $\varepsilon= \pm 1$ dépend de l'action de $\psi$ sur les orientations transverses des feuilletages); par conséquent $\psi \in G_{1}$ et $r=1$.

L'homéomorphisme $\psi$ envoie ainsi $\mu_{W}$ sur $\mu_{W}$ '. Comme ces mesures sont lisses, les feuilletages $\mathscr{F}(\mathscr{W})$ et $\mathscr{F}\left(\mathscr{W}^{\prime}\right)$ sont conjugués par un homéomorphisme $\phi \in G_{1}$ isotope à $\psi$ relativement à $F$, qui est un difféomorphisme en dehors de $F$. 
Considérons sur $M-F$ la forme différentielle $\phi(u)$. Elle est transverse à $W^{\prime}$, et on peut la modifier près de $F$ de façon à obtenir sur $M$ une forme $u^{\prime \prime}$ cohomologue à $u^{\prime}$ relativement à $F$, transverse à $\mathscr{W}^{\prime}$, avec $\mathscr{F}\left(u^{\prime \prime}\right)$ isotope à $\phi(\mathscr{F}(u))$ et égal à $\mathscr{F}\left(u^{\prime}\right)$ près de $F$.

Pour $t$ entre 0 et 1 , les formes

$$
u_{t}=(1-t) u^{\prime}+t u^{\prime \prime}
$$

sont cohomologues relativement à $F$, et non singulières en dehors de $F$ car $u^{\prime}$ et $u^{\prime \prime}$ sont toutes deux transverses à $\mathscr{W}^{\prime}$. En utilisant le résultat de Katok [3, théorème 3], ou en appliquant directement la technique d'isotopie de Moser [8], on voit alors que $\mathscr{F}\left(u^{\prime \prime}\right)$ est isotope à $\mathscr{F}\left(u^{\prime}\right)$. Il en résulte que $\mathscr{F}(u)$ et $\mathscr{F}\left(u^{\prime}\right)$ sont $G_{1}$ - conjugués.

Le théorème 2 a maintenant été ramené à l'assertion suivante:

Assertion. Etant donnée $\Omega \in A$, il existe une classe $U \in H^{1}(M, F ; \mathbb{R})$ qui contient une infinité de formes $\left(u_{i}\right)_{i \geq 1}$ telles que:

(i) si $i \neq j$, alors $\mathscr{F}\left(u_{i}\right)$ et $\mathscr{F}\left(u_{j}\right)$ ne sont pas $G_{1}$-conjugués.

(ii) pour tout $i$, il existe dans $\Omega$ une forme $\mathscr{W}_{i}$ transverse à $u_{i}$.

Pour montrer cette assertion, fixons une classe $\Omega$ dans $A$ et un disque $D \subset M$ contenant $F$. Dans $D$ une forme $\mathscr{W} \in \Omega$ s'écrit $d f$. Les valeurs de $f$ à des points différents de $F$ sont différentes, et nous pouvons écrire

$$
F=\left\{s_{1}, \ldots, s_{2 g-2}\right\},
$$

où $f\left(s_{i}\right)-f\left(s_{j}\right)$ a le même signe que $i-j$. Nous désignons par $\rho$ le nombre positif $f\left(s_{2 g-2}\right)-f\left(s_{1}\right)$.

Soient $a_{1}, \ldots, a_{g}$ des classes de $H_{1}(M ; \mathbb{Z})$ pouvant être représentées par $g$ cercles disjoints dont l'union ne sépare pas $M$. Il existe un champ de vecteurs $Y$ à singularités non dégénérées, conjugué au champ dessiné sur la figure 1 , tel que les

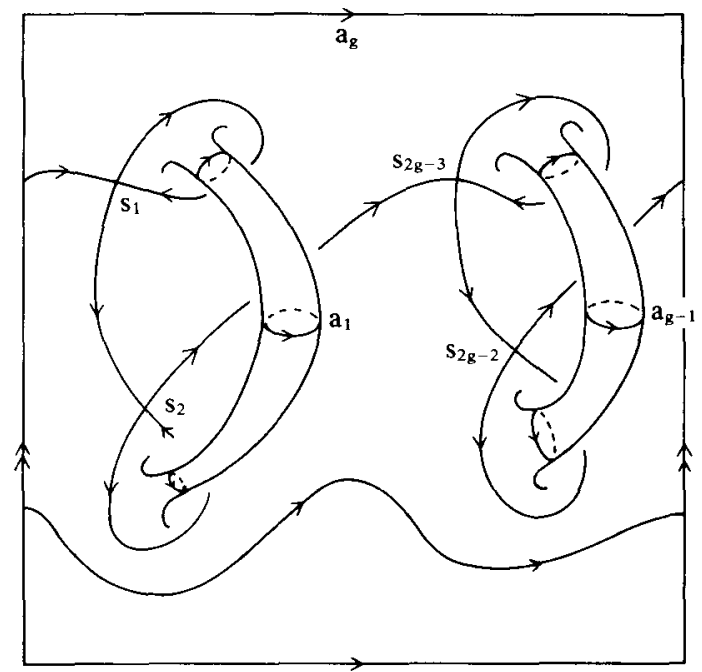

FIGURE 1. Les bords du carré doivent être identifiés deux à deux; les bords horizontaux sont des orbites de $Y$. 
liaisons $s_{i} s_{i+1}(1 \leq i \leq 2 g-3)$ soient contenues dans $D$ et que les orbites de $Y$ représentent les $a_{i}$ (comme sur la figure 1); toutes les orbites de $Y$ sont compactes, à l'exception des $4 g-4$ liaisons entres selles.

Soit $u$ une forme telle que $\mathscr{F}(u)$ soit le feuilletage déterminé par $Y$, et que la classe de cohomologie de $u$ dans $H^{1}(M ; \mathbb{R})$ soit duale de $a_{1}+\ldots+a_{g}$ (donc entière). La classe de cohomologie $U$ de $u$ dans $H^{1}(M, F ; \mathbb{R})$ est entière et ne dépend que de la somme $a_{1}+\ldots+a_{\mathrm{g}}$ (et du choix de $D$ ).

Si l'on change les classes $a_{i}$ en laissant leur somme fixe, on obtient dans la même classe $U$ des formes $u$ définissant des feuilletages qui ne sont pas $G_{1}$-conjugués. Pour déterminer lesquelles de ces formes sont transverses à une forme de $\Omega$, on utilise le lemme suivant:

LeMme 2. La classe $\Omega \in H^{1}(M, F ; \mathbb{R})$ contient une forme $\mathscr{W}$ transverse à $u$ si et seulement si $\Omega\left(a_{i}\right)>0$ pour $1 \leq j \leq g-1$ et

$$
\Omega\left(a_{g}\right)>\Omega\left(a_{1}\right)+\ldots+\Omega\left(a_{g-1}\right)+\rho .
$$

Demonstration. Si $\mathscr{W}$ existe, l'évaluation de $\Omega$ sur chacune des liaisons entre selles de $Y$ (orientées par $Y$ ) a le même signe, positif d'après le choix de la numérotation des $s_{i}$. On en déduit les inégalités désirées. Réciproquement, si ces inégalités sont vérifiées, on peut construire, dans un voisinage $Y$-invariant $E$ de l'ensemble des liaisons entre selles de $Y$, une forme fermée $\mathscr{W}_{E}$ à singularités non dégénérées, transverse à $\mathscr{F}(u)$, et définissant dans $H^{1}(E, F ; \mathbb{R})$ la même classe que $\Omega$. Chaque composante de $M-E$ est un anneau dans lequel $\mathscr{F}(u)$ est un feuilletage produit, et on peut étendre $\mathscr{W}_{E}$ dans ces anneaux en une forme $\mathscr{W} \in \Omega$ transverse à $u$.

Des classes $a_{1}, \ldots, a_{\mathrm{g}}$ de $H_{1}(M ; \mathbb{Z})$ peuvent être représentées par des cercles disjoints dont l'union ne sépare pas $M$ si et seulement si $\left\{a_{1}, \ldots, a_{\mathrm{g}}\right\}$ peut être complété en une base du $\mathbb{Z}$-module $H_{1}(M ; \mathbb{Z})$ et

$$
a_{i} \cdot a_{k}=0 \quad \text { pour tous } j, k \text {. }
$$

(- désigne la forme d'intersection.) Nous avons donc réduit le théorème 2 à l'assertion algébrique suivante:

ASSERTION. Il existe dans $H_{1}(M ; \mathbb{Z})$ une infinité de g-uplets $\left(a_{1}^{i}, \ldots, a_{\mathrm{R}}^{i}\right)_{i \geq 1}$ tels que :

(1) la somme $a_{1}^{i}+\ldots+a_{\mathrm{g}}^{i}$ ne dépend pas de $i$;

(2) pour tCus $i, j$, $k$, on $a a_{j}^{i} \cdot a_{k}^{i}=0$;

(3) pour tout $i$, on peut compléter $\left\{a_{1}^{i}, \ldots, a_{g}^{i}\right\}$ en une base de $H_{1}(M ; \mathbb{Z})$;

(4) pour tout $i$, on a $\Omega\left(a_{i}^{i}\right)>0$ pour $1 \leq j \leq g-1$, et

$$
\Omega\left(a_{\mathrm{g}}^{i}\right)>\Omega\left(a_{1}^{i}\right)+\cdots+\Omega\left(a_{\mathrm{g}-1}^{i}\right)+\rho ;
$$

(5) si $i \neq i^{\prime}$, il existe un $a_{j}^{i^{\prime}}$ qui $n^{\prime}$ appartient pas $\grave{a}\left\{a_{1}^{i}, \ldots, a_{\mathrm{g}}^{i}\right\}$.

Il nous reste à montrer cette assertion. Choisissons pour $H_{1}(M ; \mathbb{Z})$ une base $\left\{e_{i}, f_{i} ; 1 \leq i \leq g\right\}$ telle que les sous-groupes $\left(e_{i}, f_{i}\right)$ soient deux à deux orthogonaux (pour ·) et que l'évaluation de $\Omega$ sur chaque vecteur de base soit positive. Nous 
cherchons $\left(a_{1}^{i}, \ldots, a_{\mathrm{g}}^{i}\right)$ sous la forme

$$
\begin{gathered}
a_{1}^{i}=e_{1}, \ldots, a_{\mathrm{g}-2}^{i}=e_{\mathrm{g}-2}, \quad a_{\mathrm{g}-1}^{i}=x_{i} e_{\mathrm{g}-1}+y_{i} f_{\mathrm{g}-1}, \\
a_{\mathrm{g}}^{i}=-x_{i} e_{\mathrm{g}-1}-y_{i} f_{\mathrm{g}-1}+e_{\mathrm{g}}+q f_{\mathrm{g}} .
\end{gathered}
$$

( $x_{i}$ et $y_{i}$ sont des entiers relatifs, $q$ est un entier positif indépendant de $i$.)

Les conditions (1) et (2) sont automatiquement satisfaites. La condition (3) est satisfaite pourvu que $x_{i}$ et $y_{i}$ soient premiers entre eux. La condition (4) équivaut à la double inéquation

$$
0<x_{i} \Omega\left(e_{\mathrm{g}-1}\right)+y_{i} \Omega\left(f_{\mathrm{g}-1}\right)<\frac{1}{2}\left[\Omega\left(e_{\mathrm{g}}\right)+q \Omega\left(f_{\mathrm{g}}\right)-\Omega\left(e_{1}\right)-\cdots-\Omega\left(e_{\mathrm{g}-2}\right)-\rho\right] .
$$

Puisque $\Omega\left(f_{g}\right)$ est strictement positif, cette inéquation est satisfaite par une infinité de couples $\left(x_{i}, y_{i}\right)$ d'entiers premiers entre eux pourvu que $q$ soit assez grand.

\section{Exemples et questions}

(1) Soit $G_{0}$ le groupe des homéomorphismes de $M$ isotopes à l'identité relativement à $F$. Fixons $\Omega$ dans $A$. Le groupe $G_{0}$ agit sur l'ensemble des feuilletages sans liaison séparante pouvant être définis par une forme de $\Omega$, et le quotient est un ensemble dénombrable $\mathscr{H}(\Omega)$ sur lequel agit le groupe $G_{1} / G_{0}$. Le théorème 2 exprime qu'il existe une infinité d'orbites (on peut d'autre part montrer que l'action est libre). Existe-t-il un invariant simple, numérique ou autre, permettant de distinguer ces orbites?

(2) Soit $\Omega \in A$, et $\mathscr{W}$ une forme de $\Omega$ telle que $\mathscr{F}(\mathscr{W})$ ne possède pas de liaison séparante. Soit $Q \subset M$ un dodécagone bordé alternativement par des morceaux de feuilles de $\mathscr{F}(\mathscr{W})$ et des arcs transverses, comme sur la figure 2 ; les flèches représentent l'orientation transverse déterminée par $\mathscr{W}$, et les symboles $r, \delta, r+\delta$ désignent la $\mu_{w}$-mesure des intervalles auxquels ils se rapportent.

Modifions $W$ dans $Q$, comme indiqué sur la figure 2 , de manière à obtenir une forme $W^{\prime}$ cohomologue à $\mathscr{W}$ relativement à $F$. En utilisant la proposition énoncée plus haut, on peut montrer dans certains cas que $\mathscr{F}(\mathscr{W})$ et $\mathscr{F}\left(\mathscr{W}^{\prime}\right)$ ne sont pas conjugués. Il est vraisemblable qu'en fait $\mathscr{F}(\mathscr{W})$ et $\mathscr{F}\left(\mathscr{W}^{\prime}\right)$ ne sont jamais conjugués.

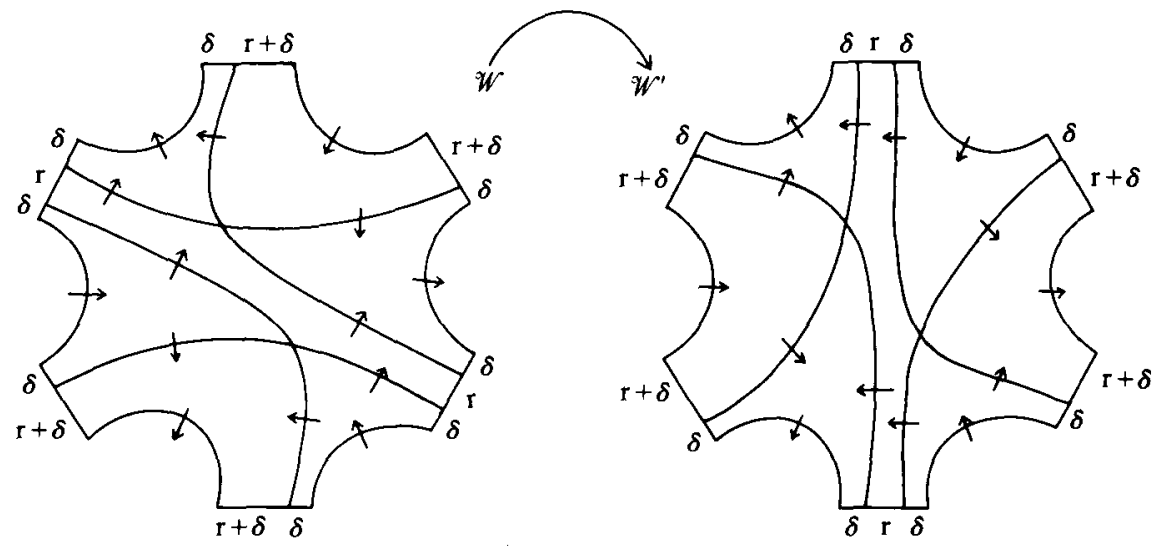

FIGURE 2. 
On peut se demander si, étant données dans une classe $\Omega \in A$ deux formes $\mathscr{W}_{1}$ et $\mathscr{W}_{2}$ telles que $\mathscr{F}\left(\mathscr{W}_{1}\right)$ et $\mathscr{F}\left(\mathscr{W}_{2}\right)$ ne possèdent pas de liaison séparante, on peut passer de l'une à l'autre par une isotopie et un nombre fini de modifications comme ci-dessus. Cette question est reliée à la suivante: considérons dans l'espace $M F$ des feuilletages mesurés de $M$ ([1], [12]) l'ensemble des feuilletages pouvant être définis par une forme appartenant à une classe de cohomologie donnée $\bar{\Omega} \in H^{1}(M ; \mathbb{R})$; cette partie est-elle connexe?

(3) Nous avons utilisé dans cet article des classes de cohomologie 'génériques' $\Omega \in A$ et des classes 'dégénérées' $U \in H^{1}(M, F ; \mathbb{Z})$. Que peut-on dire sur les classes intermédiaires? Par exemple, étant donnée une classe $\bar{\Omega} \in H^{1}(M ; \mathbb{R})$ qui n'est pas un multiple d'une classe entière, existe-t-il dans $\bar{\Omega}$ une forme $\mathscr{W}$ telle que $\mathscr{F}(\mathscr{W})$ ne possède pas de cycle de feuilles? Si oui, on peut poser une question analogue pour une classe $\Omega \in H^{1}(M, F ; \mathbb{R})$.

Je suis profondément reconnaissant envers A. Katok pour l'intérêt qu'il a porté à ce travail.

\section{RÉFÉRENCES}

[1] A. Fathi, F. Laudenbach \& V. Poenaru. Travaux de Thurston sur les surfaces. Astérisque. 66-67 (1979).

[2] D. Fried. Geometry of cross-sections to flows. Topology 21 (1981), 353-371.

[3] A. B. Katok. Invariant measures of flows on oriented surfaces. Soviet Math. Dokl. 14 (1973) (4), $1104-1108$.

[4] M. Keane \& G. Rauzy. Stricte ergodicité des échanges d'intervalles, Math. Z. 174 (1980), 203-212.

[5] G. Levitt. Pantalons et feuilletages des surfaces. Topology. 21 (1982), 9-33.

[6] G. Levitt. La décomposition dynamique et la différentiabilité des feuilletages des surfaces. Preprint.

[7] H. Masur. Interval exchange transformations and measured foliations. Preprint.

[8] J. Moser. On the volume elements on a manifold. Trans. Amer. Math. Soc. 120 (1965), 286-294.

[9] M. Rees. An alternative approach to the ergodic theory of measured foliations on surfaces. Ergod. Th. \& Dynam. Sys. 1 (1981), 461-488.

[10] S. Schwartzman. Asymptotic cycles. Ann. of Math. 66 (1957), 270-284.

[11] D. Sullivan. Cycles for the dynamical study of foliated manifolds and complex manifolds. Invent. Math. 36 (1976), 225-255.

[12] W. P. Thurston. On the geometry and dynamics of diffeomorphisms of surfaces, I. Preprint: Princeton University.

[13] W. A. Veech. Quasiminimal invariants for foliations of orientable closed surfaces. Preprint. Rice University.

[14] W. A. Veech, Gauss measures for transformations on the space of interval exchange maps. Ann. of Math. 115 (1982), 201-242. 